\section{Die Erzeugung von Röntgenblitz-Interferenzen an polykristallinem Material}

Von Werner $\mathrm{S} c \mathrm{~h}$ a a f $\mathrm{fs}$

Werkstoff-Hauptlaboratorium der Siemens \& Halske AG

(Z. Naturforschg. 5 a, 631-632 [1950]; eingeg. am 6. Nov. 1950)

Das gemeinsame Kennzeichen aller Feinstrukturaufnahmen mit Röntgenstrahlen war bisher die lange Belichtungszeit, der die photographische Platte ausgesetzt werden mußte, um eine hinreichende Schwärzung zu erzielen. Die Untersuchung einmaliger Vorgänge, die sich in ultrakurzen Zeiten der Größenordnung 10-6 sec abspielen, war nicht möglich. Sie gelang erstmals $\mathrm{Schall}$ durch Anwendung stromstarker Röntgenblitzröhren ${ }^{1}$. Mit zwei

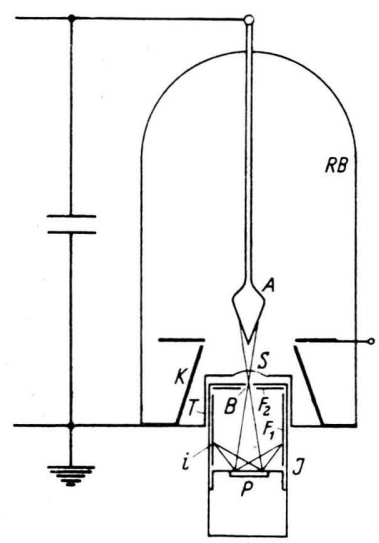

Abb. 1. Schema einer Röntgenblitzröhre mit Tauchfenster und zylindrischer Interferenzkammer. Entfernung Fokus-Präparat $3,5 \mathrm{~cm}$.

verschiedenen Methoden konnte er an gut ausgebildeten Kristallen mit einem einzigen Röntgenblitz Feinstrukturaufnahmen erhalten. Wesentlich schwieriger, aber für die physikalische Forschung und ihre technische Anwendung wichtiger, scheint es, den gleichen Effekt mit einem einzigen Röntgenblitz an polykristallinem Material $\mathrm{zu}$ verwirklichen. Von den verschiedenen besonderen Problemen, die mit einer solchen Methode bearbeitet werden können, sei nur die Erfassung kurzlebiger kristalliner Modifikationen an Phasenumwandlungspunkten genannt. Gewöhnliche Feinstrukturaufnahmen können natürlich mit der Röntgenblitzröhre auch gemacht werden; die erforderliche Expositionszeir ist um Größenordnungen kleiner.

Die ersten Versuche wurden nach dem Schema der Abb. 1 mit dem Röntgenrückstrahlverfahren an Aluminium, Messing und Steinsalz ausgeführt und ergaben, daß mindestens 5 Röntgenblitze zur Erzielung eines Diagramms notwendig waren. Zur Anwendung gelangte eine Röntgenblitzröhre, die im Prinzip schon früher beschrieben 2 , aber inzwischen weiter durchgebildet worden war.

1 R. S c h a 11, Z. angew. Physik 2, 83 [1950].

2 W. S c h a f f s, Z. angew. Physik 1, 462 [1949], Abb. 11 u. 12.
Die zylindrisch ausgebildete Interferenzkammer J wurde so weit in ein Tauchfenster $\mathrm{T}$ mit Cellophanverschluß S im Hohlkegel der Kathode $\mathrm{K}$ hineingeschoben, wie es die Entladungsbedingungen zwischen Anode A und Kathode $\mathrm{K}$ der Röhre RB zuließen. Aus dem von der Anode A aus Kupfer abgestrahlten Röntgenblitz der Kupfer-K-Strahlung wird durch eine Lochblende B ein divergierendes Strahlenbündel ausgeblendet und fällt auf das Präparat P. Einige Interferenzlinien des Rückstrahldiagramms erscheinen auf dem zylindrischen Film $F_{1}$ beispielsweise bei i. Zur Erfassung letzter Linien in Gestalt von Inter-

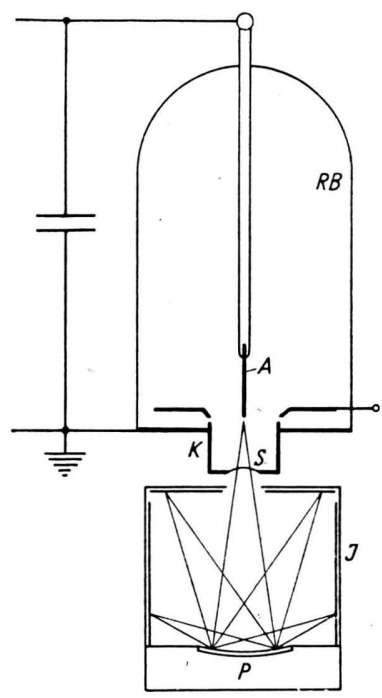

Abb. 2. Schema einer Röntgenblitzröhre mit stiftförmiger Anode und freistehender Interferenzkammer. Entfernung Fokus-Präparat $6 \mathrm{~cm}$.

ferenzringen legt man einen ebenen Film bei $\mathrm{F}_{2}$ in den Boden der Kammer. Zur scharfen Erfassung aller Linien eignet sich eine in das Tauchfenster eingeschobene Ringfilmkammer 3 .

Da die Intensität und Dauer der Röntgenblitze nicht ausreichten, um mit einem einzigen Blitz ein erkennbares Feinstrukturdiagramm zu erzeugen, und aus verschiedenen Gründen auch kaum gesteigert werden konnten, wurde ein anderes Prinzip der Erzeugung der Röntgenblitzinterferenzen erprobt. Dieses Prinzip beruht in einer Zusammenlegung von Anodenfokus und Strahlenblende. Es läßt sich experimentell, so wie es Abb. 2 zeigt, durch eine stiftförmige A ore A und durch Fortlassung aller strahlbegrenzenden B.erıden oder Spalte realisieren. Die Schärfe der Interferenzlinien hängt wesentlich von der Dicke des Anodenstiftes ab. Bei dieser Anordnung von Elektroden und Interferenzkammer steht die volle Intensität des von der Anode A in den Raumwinkel mit der Grundfläche des Präparates P abgestrahlten Röntenblitzes zur Erzeugung von Diagrammen zur Verfügung. Man kann daher mit der in Abb. 2 gezeigten Zylinderkammer J bei einer Anodenspannung von $80-90 \mathrm{kV}$ und einem sehr guten Hoch-

3 Fr. R e g l e r, Z. techn. Physik 24, 291 [1943]. 
vakuum mit Hilfe eines einzigen Röntgenblitzes an geeigneten Stoffen ein Interferenzdiagramm erzeugen.

Bei großer Intensität der Röntgenblitze und günstiger Gestaltung des Strahlenfokus 4 kann man bei einiger Übung im Dunkeln die Erscheinung subjektiv beobachten. $\mathrm{Zu}$ diesem Zweck bringt man nach Abb. 3 vor einer gewöhnlichen Röntgenblitzröhre mit kegelförmiger Anode A aus Kupfer und einem Strahlenaustrittsfenster $S$ aus Cellophan das Präparat P, beispielsweise aus Aluminium, an und beobachtet auf dem Leuchtschirm L die allerdings durch die Breite des Fokus $(2-3 \mathrm{~mm})$ verschwommenen

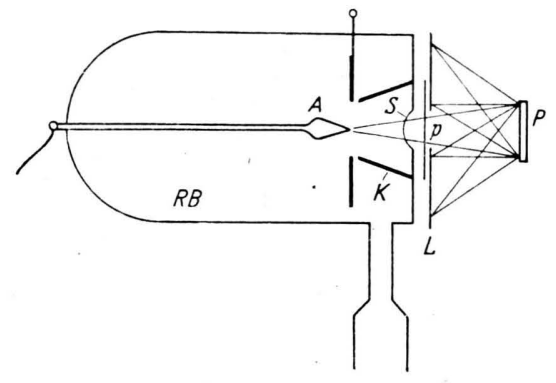

Abb. 3. Schema einer Anordnung zur subjektiven Beobachtung von Röntgenblitzinterferenzen.

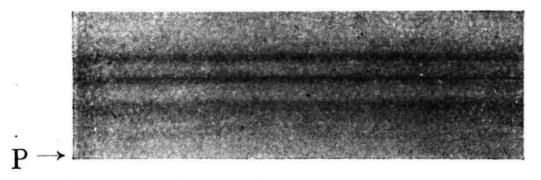

Abb. 4. Interferenzdiagramm an Aluminium, aufgenommen mit der Anordnung nach Abb. 2. Die beiden oberen Linien gehören zusammen und rühren von der $\mathrm{Cu}-\mathrm{K}_{\alpha}-$ und $\mathrm{Cu}-\mathrm{K}_{\beta}$-Strahlung her $(\mathrm{P}=$ Präparatrand $)$.

Interferenzringe mit dem Auge. Zur Fernhaltung des sichtbaren Lichtes der Entladung dient wie immer ein Blatt schwarzes Papier p.

Die Abb. 4 zeigt eine mit der Zylinderkammer nach Abb. 2 gemachte Aufnahme an einer Platte aus Aluminium. Es sind zwei Beugungsordnungen der beiden Linien der Kupfer-K-Strahlung zu erkennen. Die untere Gruppe ist verschwommen, da für sie die Fokussierungsbedingung in dieser Zylinderkammer nicht genügend erfüllt ist.

4 Vgl. W. S cha aff s u. F. Trendelen b u r g, Z. Naturforschg. 3a, 656 [1948], Abb. 2; W. S c h a a f f s, Z. angew. Physik 1, 462 [1949], Abb. 14 c.

\section{Über eine praktische Aufstellung eines normal ausgerüsteten Polarographen}

Von O.-E. S c hw e c k e n d i e k*

(Z. Naturforschg. 5a, 632—633 [1950]; eingeg. am 4. Nov. 1950)

Es wird über einen praktischen Aufbau eines handelsüblichen Polarographen berichtet. Da die polarographischen Analysenmethoden immer mehr Eingang in die
Laboratorien finden, dürfte diese Beschreibung eine kleine Anregung für die Praxis sein.

Die seit Kriegsende auf dem deutschen Markt erhältlichen „Polarographen“ 1 haben für die Praxis hinsichtlich ihrer Aufstellung noch Nachteile. Besonders die feste Anordnung von Spiegelgalvanometer und Beleuchtungseinrichtung an der Laborwand ist oft unbequem. Es wurde daher ein brauchbarer Zusammenbau entwickelt, welcher sich als sehr vorteilhaft für polarographische Arbeiten erwiesen hat. Man erhält ein transportables Gerät, welches den Raum eines gewöhnlichen fahrbaren

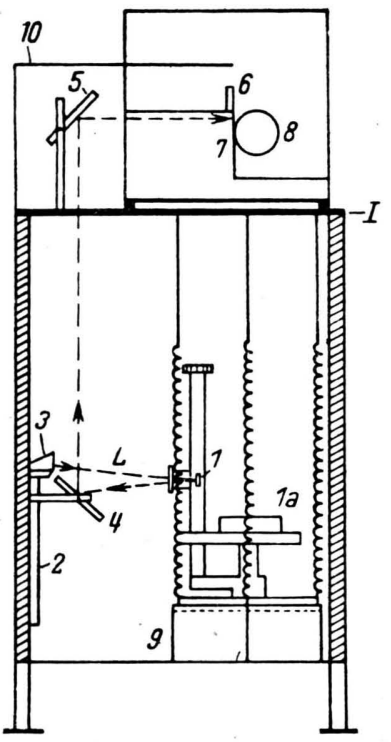

Abb. 1. Spiegelgalvanometer mit eingebauter Beleuchtungseinrichtung (Bezeichnungen s. Text).

Labortisches von etwa $75 \times 45 \times 80 \mathrm{~cm}$ beansprucht. Hierüber soll im folgenden kurz berichtet werden.

Das Galvanometer, die Beleuchtungseinrichtung, der Polarograph und die $\mathrm{Hg}$-Tropfelektrode sind $\mathrm{zu}$ einem Ganzen zusammengefaßt.

Wie aus Abb. 1 zu ersehen ist, wird der Galvanometerspiegel 1 von dem Lichtstrahl der Beleuchtungseinrichtung 2 über das drehbare und feststellbare Prisma 3 beleuchtet, durch die richtige Neigung der spiegelnden Fläche des Prismas erreicht man, daß der vom Galvanometerspiegel reflektierte Lichtstrahl so auf den ebenfalls um die waagerechte Achse drehbar angeordneten Spiegel 4 fällt und daß er von dort unter dem richtigen Winkel den Spiegel 5 trifft und von diesem auf die Skala 6 und den Aufnahmespalt 7 der Filmtrommel 8 reflektiert wird.

Durch Hin- und Herverschieben der Beleuchtungseinrichtung kann man eine scharfe Abbildung des Lichtzeigers auf der Beobachtungsskala und damit auch auf der Filmtrommel leicht erreichen.

Das Galvanometer $1 \mathrm{a}$ ist an dem oberen Auflagebrett I

* Eystrup a. d. Weser, Behring-Institut.

1 Firma Ley bold, Köln. 